\title{
Mucoid Staphylococcus aureus isolated from a patient with a suspected urinary tract infection
}

We report the isolation of an unusual morphological mucoid variant of Staphylococcus aureus. An 82-year-old male presented to the Urology Department at Belfast City Hospital with a suspected urinary tract infection, for which he submitted a midstream urine (MSU) sample. Bacteriological culture was performed on a commercial chromogenic culture medium for the culture of urinary pathogens (CPS ID 3 agar; bioMérieux) at $37{ }^{\circ} \mathrm{C}$ for $24 \mathrm{~h}$, and revealed the presence of a mucoid organism, with the typical appearance of a coliform. Subsequent Gram-staining revealed the presence of a Gram-positive coccus, which was further characterized by standard phenotypic assays (Brown et al., 2005) as S. aureus. The identification of this isolate was confirmed by employment of the BBL Crystal Gram-positive identification system, which gave a profile of 0064673045, indicating S. aureus (85\% identity). Given this relatively low percentage identity, the isolate was characterized molecularly through employment of broad range $16 \mathrm{~S}$ rDNA PCR and automated sequence-based analysis, as described previously (Xu et al., 2004). This confirmed the isolate was $S$. aureus ( $100 \%$ identity in 940 bases); the sequence was subsequently submitted to GenBank with accession number EU305717 and the isolate was archived in the culture collection of the Northern Ireland Public Health Laboratory. On routine antibiotic disc susceptibility testing, in accordance with the Clinical and Laboratory Standards Institute criteria, the isolate was resistant to penicillin and trimethoprim, but was sensitive to all other antibiotics tested, including augmentin, ciprofloxacin, clindamycin, erythromycin, fusidic acid, gentamicin, rifampicin, teicoplanin, tetracycline and vancomycin. The organism had an oxacillin MIC of $1.0 \mu \mathrm{g} \mathrm{ml}^{-1}$. The mucoid nature of the organism was present following multiple

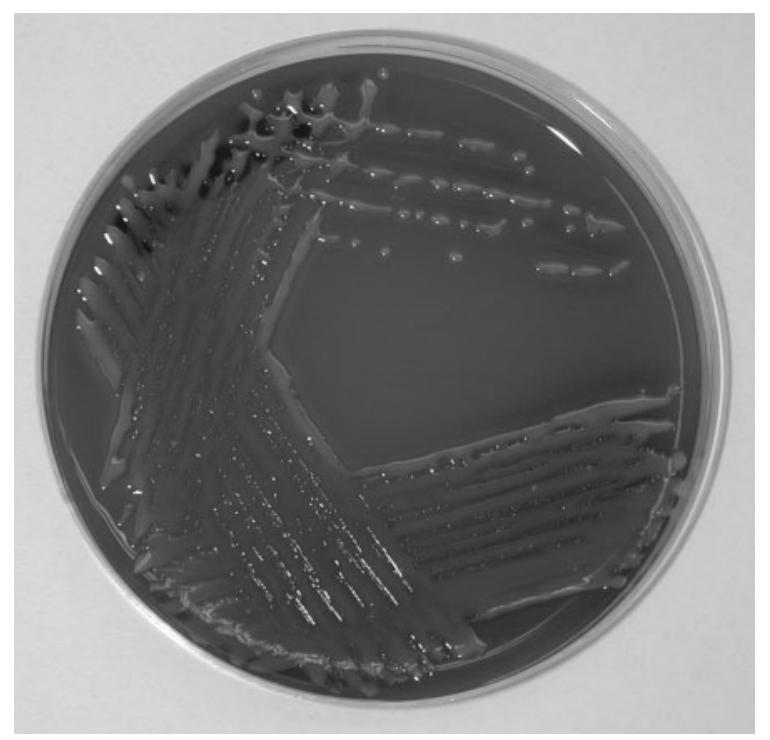

passages on Columbia blood agar supplemented with $5 \%(\mathrm{v} / \mathrm{v})$ horse blood, as shown in Fig. 1. Although rare in everyday clinical microbiology, the existence of wild-type mucoid variants of S. aureus has been described by other authors (Lee et al., 1987), where the mucoid nature was attributed to the production of capsular polysaccharides, which also has been described as being an important virulence factor in organisms with capsulated polysaccharides. (For a comprehensive review see O'Riordan \& Lee, 2004.) The enhanced virulence of encapsulated bacteria is generally attributed to resistance to phagocytosis, and published studies have demonstrated that encapsulated strains of $S$. aureus are more virulent in mice than strains lacking capsules (Sompolinsky et al., 1985;

Yoshida \& Ekstedt, 1968). However, in the patient described above, no further MSU sample or other clinical specimens were submitted for bacteriological examination, suggesting the complete resolution of his clinical condition. Therefore, although extremely rare, clinical bacteriologists should be aware of the existence of mucoid variants of $S$. aureus originating from clinical specimens and be careful colonies of not to confuse colonies of such organisms with coliforms.

Jonathan Stirling, Yasunori Maeda, B. Cherie Millar, Robert B. McClurg, Paul J. Rooney, Anne Loughrey, Colin E. Goldsmith and John E. Moore

Northern Ireland Public Health Laboratory, Department of Bacteriology, Belfast City Hospital, Lisburn Road, Belfast, BT9 7AD, Northern Ireland, UK

Correspondence: John E. Moore (jemoore@niphl.dnet.co.uk)

Brown, D. F. J., Edwards, D. I., Hawkey, P. M., Morrison, D., Ridgway, G. L., Towner, K. J. \& Wren, M. W. D. on behalf of the Joint Working Party of the British Society for Antimicrobial

Fig. 1. Mucoid S. aureus growing on Columbia blood agar. This organism was isolated from a MSU sample from an elderly male patient with a urinary tract infection. 
Infection Control Nurses Association (2005). Guidelines for the laboratory diagnosis and susceptibility testing of methicillin-resistant Staphylococcus aureus (MRSA). J Antimicrob Chemother 56, 1000-1018.

Lee, J. C., Michon, F., Perez, N. E., Hopkins, C. A. \& Pier, G. B. (1987). Chemical characterization and immunogenicity of capsular polysaccharide isolated from mucoid Staphylococcus aureus. Infect Immun 55, 2191-2197.
O’Riordan, K. \& Lee, J. C. (2004). Staphylococcus aureus capsular polysaccharides. Clin Microbiol Rev 17, 218-234.

Sompolinsky, D., Samra, Z., Karakawa, W. W., Vann, W. F., Schneerson, R. \&

Malik, Z. (1985). Encapsulation and

capsular types in isolates of Staphylococcus aureus from different sources and relationship to phage types. J Clin Microbiol 22, 828834.
Xu, J., Smyth, C. L., Buchanan, J. A., Dolan, A., Rooney, P. J., Millar, B. C., Goldsmith, C. E. Elborn, J. S. \& Moore, J. E. (2004). Employment of $16 \mathrm{~S}$ rDNA gene sequencing techniques to identify culturable environmental eubacteria in a tertiary referral hospital. $J$ Hosp Infect 57, 52-58.

Yoshida, K. \& Ekstedt, R. D. (1968). Relation of mucoid growth of Staphylococcus aureus to clumping factor reaction, morphology in serumsoft agar, and virulence. J Bacteriol 96, 902-908. 